\title{
The potential of nitisinone for the treatment of Alkaptonuria
}

Adam M Taylor ${ }^{1}$ BSc, PhD, Laura Shepherd ${ }^{1}$.

$\underline{\text { Job titles }}$

Adam Taylor $=$ Professor of Anatomy

Laura Shepherd $=$ Medical Student

${ }^{1}$ Lancaster Medical School, Lancaster University, Lancaster, UK.

Tel: 00441524593131

Email: a.m.taylor@lancaster.ac.uk 


\section{Abstract}

Introduction: Alkaptonuria is an iconic disease, dating back to the Egyptians and has continued to prove a valuable teaching tool to many medics as an example of an inborn error of metabolism. In recent years, much progress has been made on understanding the condition and its symptoms; the single defective gene has been identified and cloned. In humans novel phenotypic presentations of the condition have been documented, and in vitro models developed to understand the disease. Furthermore, a mouse model has studied and most recently clinical trials into the effectiveness of nitisinone have been undertaken. Nitisinone has been on a miraculous journey from its discovery as a weed killer to its effective treatment in hereditary tyrosinaemia type -1 .

Areas covered: The authors describe research into nitisinone and its application in model and human systems in both hereditary tyrosinaemia type 1 (HTT-1) and Alkaptonuria. The published literature was searched for outputs relating to "Alkaptonuria", "ochronosis", "nitisinone", "hereditary tyrosinaemia type 1 ". Any paper not in English or had no translation were excluded.

Expert opinion: Now nitisinone is being studied for use in Alkaptonuria and this paper documents the journey of AKU and the promising potential of nitisinone in the treatment of Alkaptonuria.

\section{Introduction}

Alkaptonuria (AKU) (OMIM: 203500) is a rare disease that currently has no approved therapy. The condition does not lead to a shortened life, but it has a significant effect on quality of life of those with the disease [1]. Over 100 years ago, Sir Archibald Garrod used AKU to illustrate the concept of Mendelian inheritance, owing to the autosomal recessive nature of AKU [2], it became a disease of both historic and medical interest. The condition is classified as rare or ultra-rare; its prevalence is documented at approximately 1:100,000-1:250,000, with hotspots reported in the Dominican Republic and Slovakia where its prevalence increases to approximate $1: 19,000$ [1]. AKU is a metabolic disease of tyrosine catabolism, caused by a deficiency or a defect of the enzyme homogentisate 1,2-dioxygenase (HGD) (EC number: 1.13.11.5) that catabolises homogentisic acid (HGA) [3]. This catabolism opens the benzene ring of the HGA molecule to the linear maleylacetoacetic acid. The HGD enzyme is believed to be highly specific in its action and is produced mainly by hepatocytes in the liver and is present in the kidneys $[1,4]$. In the absence or defect of the HGD enzyme, HGA accumulates systemically; in the cells and tissues it polymerises to benzoquinone acetic acid which pigments and turns black, a process known as ochronosis [5-7]. This process has some similarities to the conversation of tyrosine to melanin. Ochronosis is one of the features of 
the clinical triad of AKU and leads to systemic ochronosis, over time, of multiple collagenous tissues leading sufferers to progress to having ochronotic osteoarthropathy [8]. The signs and symptoms of this clinical triad associated with AKU occur sequentially. The first detectable sign of $A K U$, and the only symptom present in the paediatric population, is black or dark urine. This symptom is clearly seen in the paediatric population as it is visible in the nappy, but can often be mis-diagnosed due to the rarity of the condition and the lack of familiarity with it and its symptoms by healthcare practitioners. This darkening is the result of the oxidation of excess HGA that has been excreted via the renal system [9]. Ochronosis appears to occur as the renal system struggles to clear the HGA and so the excess HGA remaining in the body appears to accumulate in the connective tissues of the body where it undergoes polymerisation [1]. As well as in connective tissues of the musculoskeletal system, ochronosis can also occur in the eyes, ears, renal system, skin and cardiovascular system $[4,8,10]$. Ochronotic osteoarthropathy, the final feature of the triad, occurs due to the cumulative effect of the HGA polymer deposition in the collagenous connective tissues, specifically the hyaline cartilages of weight bearing joints [1]. The pigmentation commences deep in the cartilage associated with individual chondrocytes and will spread out to the cartilage matrix [11]. The significant anatomical changes in the joint are the result of the loss of subchondral bone due to aggressive bone resorption as a result of the stiff ochronotic cartilage preventing load being applied to the subchondral bone. This is further compounded by low bone turnover due to the presence of ochronotic pigment in tissues preventing resorption. The hyaline articular cartilage also undergoes significant change, it changes mechanically due to the stiff ochronotic pigment, as well as the presence of ochronotic pigment changing its biochemical properties. All of these factors contribute to the destruction of the joint and increase the susceptibility to injury and damage as the mechanical properties of the joint are altered [12]. The most common treatment for this arthropathy will result in the patient requiring a joint replacement, particularly of the weight bearing joints (hip, knee and shoulder) [5].

Keywords: Alkaptonuria, ochronosis, nitisinone, hereditary tyrosinaemia type 1, cartilage, homogentisic acid, tyrosine.

\section{Methods}


Our manuscript includes research outputs that were generated from searching online literature databases PubMed and Google Scholar using the search terms "Alkaptonuria", "ochronosis", "nitisinone", "hereditary tyrosinaemia type 1". There were no limitations on the dates used to exclude papers. The authors only read papers that were published in English or had a freely available translation from their native language.

The authors describe in vitro and in vivo research into nitisinone and its application in model and human systems in both hereditary tyrosinaemia type 1 (HTT-1) and alkaptonuria.

\section{Potential therapies}

At present, there is no cure for AKU and the associated ochronotic osteoarthropathy, treatment/management options are limited and often palliative with a lack of curative or preventative options [1]. Physiotherapy, which is aimed at maintaining mobility and reducing the rate of musculoskeletal decline has been utilised [13]. Pain management is frequently administered, although the delivery of any analgesia can be impacted due to the ochronosis of tissues, particularly when considering the spine and shoulders [14-18]. Due to the lack of a disease modifying therapy, ultimately, multiple joint replacements are needed [19-21]. All of these only treat the symptoms, they do not deal with altering, removing or reducing the causative molecule, HGA, from the body.

A low protein diet, to limit the intake of the preceding amino acids that are broken down to HGA; tyrosine and phenylalanine has been suggested as a potential therapy for AKU patients, it is incredibly difficult to maintain and is thought to be ineffective due to this reason and the fact that less than $10 \%$ of dietary protein is broken down via the HGA pathway. It is difficult to sustain this in the long term and can be problematic during childhood as limiting intake of protein can have an effect on development but compliance may also be problematic $[22,23]$. It is also clear that not all tyrosine and phenylalanine comes from dietary intake, some is generated through tissue catabolism[22].

Anti-oxidant therapies, such as ascorbic acid (ASC) more commonly known as Vitamin-C, have been suggested as potentially inhibitors of the polyermisation of HGA, showing effectiveness in reducing the conversation of HGA to its quinone intermediate on the way to ochronotic pigment, it did not reduce the amount of HGA excreted in the urine [24]. A greater understanding of the tyrosine degradation pathway also highlights that ASC is a co-factor for 4-hydroxyphenylpyruvate dioxygenase, which causes increased HGA production by ensuring 
the conversion of 4-hydroxyphenylpyruvic acid to HGA, this may actually increase the amount of HGA formed and therefore the amount of HGA available to undergo the polymerisation process to pigmentation. Evidence of the use of ascorbic acid in the paediatric population demonstrated a concerning increase in the urinary concentration(s) of HGA which may facilitate pigmentation in earlier age groups [25]. This increase in HGA levels may also contribute to the formation of renal oxalate stones. This is concerning, as AKU patients are already at high risk for developing renal calculi and prostatic calculi in males [26-28]. The mechanism of calculi formation in AKU patients is unclear, it is documented that the development of calculi forms in a similar manner to that in the general population. However, the literature reports that there is an increase in the prevalence of any type of stones; renal, prostatic or even submandibular in the AKU population [29, 30]. Formation of oxalate stones, requires an interaction between the renal system epithelia and the crystal. Usually the crystals formed within the system are discharged in the urine, small crystals that become attached to the epithelial lining are thought to be digested by macrophages or lysosomes. Factors that initiate the formation of stones include reduced urinary volume or alterations in urinary $\mathrm{pH}$ or a combination of these factors [31]. Given that AKU patients are excreting gram quantities of HGA daily in their urine [32], this will cause perturbations in their urinary $\mathrm{pH}$ and may be a factor in the increased formations of stones in renal, or any other excretory, or secretory system in these patients. The association of ochronotic pigment and calcium crystals has previously been documented both in calculi, but also in other calcified pathological areas of the body suggesting an overlap in the process [33]. it is not ultimately clear exactly how HGA or calculi interact and further work is needed in this area to decrease the disease burden in AKU patients [34]. It has been shown that liver transplantation is a successful means to eradicate HGA from the body, due to the presence of the HGD enzyme in the donor liver hepatocytes [35]. This halted the progression of the disease, but is unlikely to have reversed the presence of any ochronosis that already existed in the tissues.

A drug under current investigation for use in AKU is nitisinone [1], an inhibitor of 4hydroxyphenlypyruavte dioxygenase, which is also present in the tyrosine degradation pathway [36]. Although nitisinone is not routinely prescribed for AKU, it is administered to patients who are being treated at the National AKU centre based in Liverpool [37]. This drug 
is licensed for treatment of hereditary tyrosinaemia type 1 , another, more severe disorder of tyrosine metabolism [38].

\section{Discovery of Nitisinone}

Nitisinone is a naturally occurring herbicide discovered when observations were made of the reduction in the number of other plants growing in close proximity to the bottlebrush plant (Callistemon) [39]. The absence of other species from around the bottlebrush suggested an effect from inhibition of pathway(s) affiliated with growth. It was discovered that the chemical known as Leptospermone, which is present in the soil around the plants had a bleaching effect, amongst others, on neighbouring plants. Leptospermone belongs to the triketone family and impedes chloroplast development by 4-hydroxyphenylpyruvate dioxygenase (HPPD) inhibition; HPPD is an enzyme involved in the catabolism of tyrosine and is integral to the production of a number of natural plant products $[40,41]$. The discovery of the triketone family, of which Leptospermone was an early finding, led to the discovery and development of nitisinone. The initial work in understanding how nitisinone worked was undertaken by Zeneca Agrochemicals following indications that it inhibited the actions of another enzyme which uses tyrosine as a substrate; tyrosine hydroxylase. Further work on nitisinone by Zeneca Central Toxicology Laboratories clarified that Nitisinone did not inhibit tyrosine hydroxylase or tyrosine aminotransferase, but in fact was a potent inhibitor of HPPD [38].

Whilst this paper is interested in the potential of nitisinone in AKU, it is another disorder of tyrosine metabolism where nitisinone would initially prove its value as a therapy. AKU is not shown to reduce longevity, however hereditary tyrosinaemia type 1 (HT-1)(OMIM \#276700) is a much more life limiting disorder which if left untreated can result in hepatocellular carcinoma and other severe complications leading to death at a young age [42]. Prior to the discovery and availability of nitisinone, it was liver transplantation that was the go to therapy for HT-1. In the HT-1 paediatric population(s), the administration of nitisinone in combination with a protein restricted diet, to individuals under 2 months of age sees the 4 year survival rate increase to $94 \%$, compared to protein controlled diet alone at $29 \%$ [43].

The ongoing work to understand the mechanism of action of nitisinone would see it administered to multiple animals in order to test its efficacy and toxicity. Administration to 
rats produced a marked increase in the presence of tyrosinaemia in plasma and the aqueous humour of the eye; $2500 \mathrm{nmol} / \mathrm{L}$ and $3500-4000 \mathrm{nmol} / \mathrm{L}$ respectively [44]. These tyrosinaemias were produced by the administration of $0.3 \mu \mathrm{mol} / \mathrm{kg} /$ day and $30 \mu \mathrm{mol} / \mathrm{kg} /$ day nitisinone, which resulted in tyrosinaemia occurrence in $38 \%$ and $75 \%$ of subjects. Whilst an increase in presence of corneal opacities as a result of tyrosinaemia was noted, the question raised is one of what systemic factors within individual subjects may contribute, or protect against this symptom? Variation in plasma concentrations of tyrosine, or abundance of enzyme(s) or their level of activity in the tyrosine metabolic pathways may act as rate limiting, and therefore, symptom limiting factors.

\section{Why is it needed in AKU?}

AKU has encountered a vast number or potential therapies, many of which have had varied success, but the bottom line has always been that there is no effective therapy, only management by palliative care such as physiotherapy or pain relief and then surgical replacement of the affected joint.

Research on HT-1 has again given hope to patients with the condition, and the hope is that it may stand to benefit patients with AKU, similar to that seen in the discovery, development and use of nitisinone. Gene-editing therapies such as those utilising the CRISPR-Cas9 have been utilised in vivo to show that this technology can rescue diseased phenotypes, such as HT-1 in adult mice [45], or convert them to a more benign phenotype [46]. Most recently this technology has shown that it can produce phenotypic rescue by utilisation in utero, again correcting the potentially lethal HT-1 phenotype [47]. The ability to edit the genetic make-up of hepatocytes in this way holds promise for other diseases of the tyrosine metabolic pathway, including diseases such as AKU. In AKU, it is known that only the HGD enzyme is missing and thus a single defect correction would be sufficient to ensure correction of disease $[48,49]$. Delivery of the functional HGD code to hepatocytes is significant here, to ensure that subsequent tyrosinaemias in the pathway are not induced based on the locality of the cytosolic production of tyrosine metabolic enzymes in the hepatocytes of the liver.

\section{Effectiveness of Nitisinone in AKU}


A positive reduction in HGA levels has been seen in associated with the use of this drug [50, 51]. However, there have been undesirable side effects including corneal opacities and limited evidence have restricted universal use to date [52]. These corneal side effects are most commonly described in HTT-1 patients at a prevalence of $7.4-8.7 \%$ based on the studies undertaken $[53,54]$. The doses of Nitisinone used in HTT-1 treatment is approximately 20 fold higher than that used in AKU and therefore the reported complications of corneal opacities in the AKU literature is much rarer [55]. Previous studies have utilised endpoints in relation to the musculoskeletal system, with hip range of motion as a primary outcome and other musculoskeletal actions as secondary measures. These parameters did not show significant enough improvement to validate the use of nitisinone for AKU [51]. The interesting data in the study demonstrated that the urinary and plasma HGA levels were reduced by at least $95 \%$ in both cases, in some instances to undetectable levels, replicating non-AKU levels [51]. The HGA lowering efficiency shown in this trail replicated the effect seen in the preliminary study demonstrating the positive effect of low dose of nitisinone on the presence of HGA in urine of a small cohort of AKU patients [56]. Most recently, a specialist NHS commissioned service gave $2 \mathrm{mg}$ of nitisinone every other day for 3 months and daily thereafter to a group of 58 patients, with 28 of these having 2 years' worth of nitisinone treatment in Liverpool, has again shown the significant effect of nitisinone on the reduction of HGA in urine and serum, with reductions approaching $95 \%$ and over $80 \%$ respectively [37]. Similar extremely positive outcomes in the SONIA-1 (Suitability of Nitisinone in Alkaptonuria) study with promising clinical results in demonstrating a $98.8 \%$ reduction in urinary excretion of HGA over 24 hours, when patients are administered 8mg/day [57]. In other work, low doses of nitisinone have also demonstrated efficacy in patients in the paediatric age group $[57,58]$.

The effect of nitisinone on HGA levels is clearly positive in terms of potential benefit to patients. It has the ability to reduce ochronosis by removing or reducing the causative molecule HGA from the body. Over time the polymerisation of HGA has many known and potentially unknown effects - the removal of the HGA molecule altogether ameliorates any issues that may arise due to its presence. This is ideal in terms of reducing disease burden for the patient and returning to a normal quality of life across the lifespan [59]. The discovery of a murine model which presents with the AKU genotype and phenotypic symptoms similar to the human has aided in the understanding of the disease process and the effectiveness of 
nitisinone [60]. Whilst the only symptom of AKU visible in the paediatric age group is the homogentisic aciduria, the timing of manifestation of ochronosis in tissues is still unclear, but appears to be around the $3^{\text {rd }}$ or $4^{\text {th }}$ decade. The mouse has proved useful in demonstrating that the pigmentation process commences around week 15 of life and that nitisinone arrests, but does not reverse ochronosis [50,61].

Little is known about the systemic effects of nitisinone in humans, however, it has been shown to have no adverse effects on osteoarticular cells, which without the presence of nitisinone in AKU are subject to intra and extracellular ochronosis $[62,63]$. This all provides promising evidence for the use of nitisinone and the reduction of HGA, limiting the ochronosis of tissues.

The main side-effect of administration of nitisinone is the accompanying tyrosinaemia that develops in some of the patients due to the increase in the presence of plasma tyrosine levels. The most concerning side effect that has been seen in individuals with tyrosinaemia is the potential for cognitive impairment in terms of development and beyond. In the early studies that examined this in HTT-1 patients, there were suggestions that approximately $35 \%$ of school age children had some form of difficulties [54]. However, this study lacked what are now considered routine psychometric tests. Further studies have identified variation in the HTT-1 population in terms of their cognitive function with one of the limiting factors being the small number of patients that were present in each of the studies [64]. The withdrawal of nitisinone or non-compliance has other complications such as neurological crises $[42,65]$. There are several proposed mechanisms for the potential neurological developmental delay that is seen in HTT-1, they all relate to movement of monoamine molecules such as tyrosine and tryptophan into the brain, as well as other more peripheral or central nervous system effects [66-68]. It is important in the study of AKU in particular to understand when the most optimal treatment time for commencing nitisinone is. Whilst the information of the effect of HGA on the body in the paediatric age group is limited, it does not appear to have the detrimental effects that it, or the subsequent polymer has in the adult population. Similarly, if tyrosinaemias associated with the administration of nitisinone in the paediatric population of HTT-1 patients produce cognitive issues in development a decision has to be made as to when would be appropriate to commence nitisinone therapy in AKU. Further work and understanding in this area is needed, but data from the AKU mouse suggests that monoamine neurotransmitters in the brain tissues of AKU mice did not change following the 
administration of nitisinone [68]. The increased plasma tyrosine causes retinal keratopathy and/or corneal opacities in the eyes of AKU patients caused by the deposition of tyrosine crystals $[55,69,70]$. The presence of tyrosine crystal deposits in the ocular tissues is not fully understood, however cessation of nitisinone results in disappearance of crystals and symptoms as soon as 2 weeks after halting therapy, although deposition may still occur even in the absence of symptoms and this should be monitored closely. The monitoring of the levels of tyrosine and HGA are key indicators to assess efficacy of nitisinone therapy in patients administered nitisinone in pre- and post-administration and to ensure that patient safety is maintained, this has been benefitted by the development of techniques to assess both HGA and tyrosine simultaneously in urine samples [71]. This technique has been further enhanced to measure nitisinone levels in combination with HGA and tyrosine within serum samples [30].

The most recent clinical trial examining the effect of nitisinone in AKU patients: Suitability of Nitisinone in Alkaptonuria 2 (SONIA 2) has shown that patients treated for 3-12 months had a $99.5 \%$ reduction in the HGA they excreted in their urine and a $97.5 \%$ reduction in the HGA detectable in their blood after one year. The patients in this study tolerated nitisinone remarkably well with only 5 patients showing ocular problems and being switched to a lower dose of nitisinone. The investigators conclude that the "SONIA 2 one year study data shows that nitisinone treatment of AKU patients results in a significant reduction of HGA in the urine and blood with no safety concerns." The strength and success of these results has led Swedish Orphan Biovitrium to seek market authorisation from the European Medicines Agency for the drug to be used in treating AKU [72].

Recently it has been shown that Raman spectroscopy may be a useful tool for monitoring ochronosis in vivo. The ex vivo analysis of cartilage samples from the hips and ears of individuals with AKU have shown unique cartilage spectra from those with the presence of ochronotic pigment, whilst cartilages without ochronosis demonstrated a spectra that was consistent with normal cartilages [73]. Raman has the potential to be used in vivo via transdermal analysis of cartilage, it has been used on analysing cartilage and bone samples from patients with Osetogenesis Imperfecta (OI). The technique of Raman spectroscopy has already provided useful information in a clinical setting on the composition of bone matrix via transcutaneous acquisition of osteoporosis and osteogenesis imperfecta [74, 75]. In AKU, one of the early observable tissues that undergoes ochronosis is the ears, Raman has the potential 
to be used for real-time analysis of this tissue, as well as the longitudinal monitoring of patients who may at some point need nitisinone administration. If spectral analysis of tissues demonstrated the presence of ochronotic pigment the affected individual could be placed onto nitisinone to halt progression of the condition. Similarly, the use of Raman to monitor osteoarticular tissues, has been shown and this could also be applied to these tissues in AKU patients. Prior to the use of Raman spectroscopy clinicians and researchers utilised a robust and validated scoring index the AKU severity score index (AKUSSI) which characterised a preochronotic and then a much more rapidly progressing ochronotic state of the disease, which was known by clinicians and researchers but up until this point had been difficult to quantify and demonstrate. This tool also demonstrates the burden of the disease [76]. As AKU research has gathered traction and more patients have become involved in research, the AKUSSI has clearly demonstrated the effect of nitisinone on the various parameters of the index. It shows how effective the therapy is, adding further validation to its use in AKU, it reduces HGA but also demonstrates numerous secondary important features of monitoring patients and their tissues which become symptomatic during the course of the disease $[77,78]$. The efficacy of nitisinone is clearer seen in longitudinal studies spanning 3 years examining multiple patients with AKU, not only reducing HGA, but also arresting ocular and auricular ochronosis[59].

\section{Expert Opinion}

Nitisinone has gone a significant journey since its discovery as a weedkiller to becoming a wonder drug for treating potential fatal conditions. The early trial of the therapy in AKU was disappointing, but subsequent in vitro, animal and now clinical trial data suggest that a primary outcome measure as the reduction of HGA in plasma and urine as an end point have renewed hope for AKU patients around the world. The absence of ochronosis in mice administered nitisinone through drinking water shows a clear efficacy for the drug and correlation with the reduction of HGA resulting in the absence of pigmentation suggests that in model systems that this should be replicated in humans.

There are still challenges and research to be done to understand the variability in presentation of tyrosinaemia and ocular pathologies associated with nitisinone administration in AKU patients, it is likely there are other variable factors within individuals that are responsible for these variations given that the dose of nitisinone administered in trials affects some but not others. The validation of Raman spectroscopy as a technique that has the ability to identify 
differing spectra of tissues with and without ochronosis is an exciting proposition particularly as it has already been validated as being able to detect pathological matrix in other osteoarticular tissues. This may prove useful for monitoring the absence/presence of ochronosis and enable the therapy to be targeted to an individuals presentation of ochronosis in tissues, given there is variability within an individual's connective tissues (different tissues pigment at different rates) and across affected individuals. This may also mean that there is a reduced requirement for younger populations needing the administration of nitisinone, which still requires further research in AKU populations. AKU may also benefit from future advances in gene replacement or enzyme replacement therapies, only time will tell. The fact that this disease is the result of a single enzyme defect that causes elevation in a single tyrosine metabolite suggests that it may be a useful model to look at gene therapies for more complex conditions where some of this information is yet to be clarified.

AKU is an iconic disease due to Garrod's work and demonstrating that the disease results from inheritance. Since then the defective enzyme and gene have been identified, the causative molecule elucidated and studied and potential therapies have come and gone.

There is still work for researchers to do in trying to understand the biochemical changes that occur to HGA in the polymerisation process and how this promotes binding to extracellular matrices. These questions will need to be answered to look at removing established ochronosis from patient tissues. The current landscape suggests that any therapy that inhibits the presence of ochronosis, by removing the presence of HGA altogether, is the most efficient and sensible way forward.

At the moment the effectiveness of nitisinone looks to be a very positive therapy for an iconic condition that has benefitted from dedicated patients, advocates and researchers in recent years to make ground-breaking discoveries about the condition. The recent release of the SONIA 2 results and the move by Swedish Orphan Biovitrium to seek market authorisation from the European Medicines Agency for the use of nitisinone to treat AKU suggests that this "weedkiller to wonder drug" story may have another chapter to be written. This will see patients diagnosed with an iconic medical condition be able to receive treatment and the prospect of ochronosis affecting them disappears.

\section{References}

1. Mistry, J.B., M. Bukhari, and A.M. Taylor, Alkaptonuria. Rare Dis, 2013. 1: p. e27475. 
* A review on all aspects of AKU.

2. Garrod, A., The Croonian Lectures ON INBORN ERRORS OF METABOLISM. The Lancet, 1908. 172(4427): p. 1-7.

3. La Du, B.N., V.G. Zannoni, L. Laster, and J.E. Seegmiller, The nature of the defect in tyrosine metabolism in alcaptonuria. J Biol Chem, 1958. 230(1): p. 251-60.

4. Ffolkes, L.V., D. Brull, S. Krywawych, et al., Aortic stenosis in cardiovascular ochronosis. J Clin Pathol, 2007. 60(1): p. 92-3.

5. Phornphutkul, C., W.J. Introne, M.B. Perry, et al., Natural history of alkaptonuria. New England journal of medicine, 2002. 347(26): p. 2111-2121.

* The first short-term use of nitisinone in patients with AKU

6. Tinti, L., A.M. Taylor, A. Santucci, et al., Development of an in vitro model to investigate joint ochronosis in alkaptonuria. Rheumatology (Oxford), 2011. 50(2): p. 271-7.

* This paper demonstrated the first development of an in vitro system to study AKU

7. Fernandez-Canon, J.M., B. Granadino, D. Beltran-Valero de Bernabe, et al., The molecular basis of alkaptonuria. Nat Genet, 1996. 14(1): p. 19-24.

8. Taylor, A.M. and K.P. Vercruysse, Analysis of Melanin-like Pigment Synthesized from Homogentisic Acid, with or without Tyrosine, and Its Implications in Alkaptonuria. JIMD Rep, 2017. 35: p. 79-85.

9. Garrod, A., THE INCIDENCE OF ALKAPTONURIA : A STUDY IN CHEMICAL INDIVIDUALITY. The Lancet, 1902. 160(4137): p. 1616-1620.

10. Fisher, A.A. and M.W. Davis, Alkaptonuric ochronosis with aortic valve and joint replacements and femoral fracture: a case report and literature review. Clin Med Res, 2004. 2(4): p. 209-15.

11. Taylor, A.M., A. Boyde, P.J. Wilson, et al., The role of calcified cartilage and subchondral bone in the initiation and progression of ochronotic arthropathy in alkaptonuria. Arthritis Rheum, 2011. 63(12): p. 3887-96.

12. Taylor, A.M., M.F. Hsueh, L.R. Ranganath, et al., Cartilage biomarkers in the osteoarthropathy of alkaptonuria reveal low turnover and accelerated ageing. Rheumatology (Oxford), 2017. 56(1): p. 156-164.

** This paper demonstrates the first study of biomarkers of cartilage in AKU tissues and compares their change with $O A$ and normal tissues

13. Perry, M.B., P. Suwannarat, G.P. Furst, et al., Musculoskeletal findings and disability in alkaptonuria. J Rheumatol, 2006. 33(11): p. 2280-5.

14. Kalevski, S.K., D.G. Haritonov, and N.A. Peev, Alcaptonuria with lumbar disc prolapse: case study and review of the literature. Spine J, 2007. 7(4): p. 495-8.

15. Rana, A.Q., U. Saeed, and I. Abdullah, Alkaptonuria, more than just a mere disease. Journal of Neurosciences in Rural Practice, 2015. 6(2): p. 257-+.

16. Bilgi, K., S. Jagadeeshan, and P. Venugopal, Difficult epidural in a patient with undiagnosed alkaptonuria. Indian J Anaesth, 2016. 60(7): p. 523-5.

17. Kozanhan, B., [Anesthetic management of two patients with alkaptonuric ochronosis for total knee arthroplasty]. Rev Bras Anestesiol, 2018. 68(3): p. 307-310.

18. Gupta, P.K., A. Acharya, D. Sabat, and A. Mourya, Arthroscopic diagnosis and treatment of shoulder ochronotic arthropathy - A case report. J Clin Orthop Trauma, 2017. 8(Suppl 1): p. S80-S83.

19. Harun, M., Y. Hayrettin, M. Serhat, et al., A rare cause of arthropathy: An ochronotic patient with black joints. Int J Surg Case Rep, 2014. 5(8): p. 554-7.

20. Demir, S., Alkaptonuric ochronosis: a case with multiple joint replacement arthroplasties. Clin Rheumatol, 2003. 22(6): p. 437-9.

21. Spencer, J.M.F., C.L.M.H. Gibbons, R.J. Sharp, et al., Arthroplasty for ochronotic arthritis - No failure of 11 replacements in 3 patients followed 6-12 years. Acta Orthopaedica Scandinavica, 2004. 75(3): p. 355-358. 
22. de Haas, V., E.C. Carbasius Weber, J.B. de Klerk, et al., The success of dietary protein restriction in alkaptonuria patients is age-dependent. J Inherit Metab Dis, 1998. 21(8): p. 791-8.

* This paper has some of the first data showing the effect of dietary restriction of protein in AKU and the mixed efects and issues that arise.

23. Ranganath, L.R., J.C. Jarvis, and J.A. Gallagher, Recent advances in management of alkaptonuria (invited review; best practice article). J Clin Pathol, 2013. 66(5): p. 367-73.

24. Sealock, R.R., M. Galdston, and J.M. Steele, Administration of Ascorbic Acid to an Alkaptonuric Patient. 1940. 44(2): p. 580-583.

25. Wolff, J.A., B. Barshop, W.L. Nyhan, et al., Effects of ascorbic acid in alkaptonuria: alterations in benzoquinone acetic acid and an ontogenic effect in infancy. Pediatr Res, 1989. 26(2): p. 140-4.

26. Lorenzini S, M.A., Selvi E. , Alkaptonuria. 2003. 348(14): p. 1408-1408.

27. Forslind, K., F.A. Wollheim, B. Akesson, and U. Rydholm, Alkaptonuria and ochronosis in three siblings. Ascorbic acid treatment monitored by urinary HGA excretion. Clin Exp Rheumatol, 1988. 6(3): p. 289-92.

28. Masoud, H.M.F., H.H. Alhawari, N.T. Alryalat, et al., A rare presentation of alkaptonuria: Extensive prostatic calculi with highlight of stones found in a unique paraprostatic urethral diverticulum. International Journal of Surgery Case Reports, 2017. 38: p. 192-195.

29. Taylor, A.M., P.J. Wilson, D.R. Ingrams, et al., Calculi and intracellular ochronosis in the submandibular tissues from a patient with alkaptonuria. J Clin Pathol, 2010. 63(2): p. 186-8.

30. Hughes, A.T., A.M. Milan, A.S. Davison, et al., Serum markers in alkaptonuria: simultaneous analysis of homogentisic acid, tyrosine and nitisinone by liquid chromatography tandem mass spectrometry. Ann Clin Biochem, 2015. 52(Pt 5): p. 597-605.

** This paper highlights the validity and accuracy of LC tandem MS in simultaneously analysing serum HGA, tyrosine and nitisinone in AKU patients, the balance of these three comoounds is significant in ensuring appropriate management of individual patients HGA load and potential complications with being on nitisinone.

31. Coe, F.L., J.H. Parks, and J.R. Asplin, The pathogenesis and treatment of kidney stones. N Engl J Med, 1992. 327(16): p. 1141-52.

32. Aquaron, R., Alkaptonuria: a very rare metabolic disorder. Indian J Biochem Biophys, 2013. 50(5): p. 339-44.

33. Helliwell, T.R., J.A. Gallagher, and L. Ranganath, Alkaptonuria--a review of surgical and autopsy pathology. Histopathology, 2008. 53(5): p. 503-12.

34. Tsujihata, M., Mechanism of calcium oxalate renal stone formation and renal tubular cell injury. Int J Urol, 2008. 15(2): p. 115-20.

35. Kobak, A.C., G. Oder, S. Kobak, et al., Ochronotic arthropathy: disappearance of alkaptonuria after liver transplantation for hepatitis B-related cirrhosis. J Clin Rheumatol, 2005. 11(6): p. 323-5.

* An interesting case report highlihgting the effects of liver transplantation on HGA levels in an AKU patient.

36. Ranganath, L.R., O.G. Timmis, and J.A. Gallagher, Progress in Alkaptonuria--are we near to an effective therapy? J Inherit Metab Dis, 2015. 38(5): p. 787-9.

37. Milan, A.M., A.T. Hughes, A.S. Davison, et al., The effect of nitisinone on homogentisic acid and tyrosine: a two-year survey of patients attending the National Alkaptonuria Centre, Liverpool. Ann Clin Biochem, 2017. 54(3): p. 323-330.

** The first longitudinal study of administration of nitisinone in an NHS setting over a 2 year period.

38. Lock, E., L.R. Ranganath, and O. Timmis, The Role of Nitisinone in Tyrosine Pathway Disorders. J Current Rheumatology Reports, 2014. 16(11): p. 457.

* This paper summarises the journey nitisinone has taken from discovery to clincial application. 
39. Lock, E.A., M.K. Ellis, P. Gaskin, et al., From toxicological problem to therapeutic use: The discovery of the mode of action of 2-(2-nitro-4-trifluoromethylbenzoyl)-1,3-cyclohexanedione (NTBC), its toxicology and development as a drug. 1998. 21(5): p. 498-506.

40. Das, A.M., Clinical utility of nitisinone for the treatment of hereditary tyrosinemia type-1 (HT1). The application of clinical genetics, 2017. 10: p. 43-48.

41. Schenck, C.A. and H.A. Maeda, Tyrosine biosynthesis, metabolism, and catabolism in plants. Phytochemistry, 2018. 149: p. 82-102.

* This article gives a comprehensive coverage of the role of tyrosine in production of plant products and pathways.

42. Chinsky, J.M., R. Singh, C. Ficicioglu, et al., Diagnosis and treatment of tyrosinemia type I: a US and Canadian consensus group review and recommendations. Genetics In Medicine, 2017. 19: p. 1380.

43. Malik, S., S. NiMhurchadha, C. Jackson, et al., Treatment Adherence in Type 1 Hereditary Tyrosinaemia (HT1): A Mixed-Method Investigation into the Beliefs, Attitudes and Behaviour of Adolescent Patients, Their Families and Their Health-Care Team. JIMD reports, 2014. 18: p. 13-22.

44. Lock, E.A., P. Gaskin, M.K. Ellis, et al., Tissue Distribution of 2-(2-Nitro-4trifluoromethylbenzoyl)cyclohexane-1,3-dione (NTBC): Effect on Enzymes Involved in Tyrosine Catabolism and Relevance to Ocular Toxicity in the Rat. Toxicology and Applied Pharmacology, 1996. 141(2): p. 439-447.

45. Yin, H., W. Xue, S. Chen, et al., Genome editing with Cas9 in adult mice corrects a disease mutation and phenotype. Nature Biotechnology, 2014. 32: p. 551.

46. Pankowicz, F.P., M. Barzi, X. Legras, et al., Reprogramming metabolic pathways in vivo with CRISPR/Cas9 genome editing to treat hereditary tyrosinaemia. Nature Communications, 2016. 7: p. 12642.

47. Rossidis, A.C., J.D. Stratigis, A.C. Chadwick, et al., In utero CRISPR-mediated therapeutic editing of metabolic genes. Nature Medicine, 2018. 24(10): p. 1513-1518.

48. Usher, J.L., D.B. Ascher, D.E. Pires, et al., Analysis of HGD Gene Mutations in Patients with Alkaptonuria from the United Kingdom: Identification of Novel Mutations. JIMD Rep, 2015. 24: p. 3-11.

* This paper shows the genetic heterogeneity in AKU and the value of applying computational approaches to understanding the activity of the protein complex produced.

49. Beltran-Valero de Bernabe, D., B. Granadino, I. Chiarelli, et al., Mutation and polymorphism analysis of the human homogentisate 1, 2-dioxygenase gene in alkaptonuria patients. Am J Hum Genet, 1998. 62(4): p. 776-84.

50. Preston, A.J., C.M. Keenan, H. Sutherland, et al., Ochronotic osteoarthropathy in a mouse model of alkaptonuria, and its inhibition by nitisinone. Ann Rheum Dis, 2014. 73(1): p. 284-9.

** This paper is the first demonstration of the effect of nitisinone in a model animal system showing plasma concentrations and correlations with ochronosis.

51. Introne, W.J., M.B. Perry, J. Troendle, et al., A 3-year randomized therapeutic trial of nitisinone in alkaptonuria. Mol Genet Metab, 2011. 103(4): p. 307-14.

** The first randomized trial using nitisinone in AKU patients with results showing primary outcome as not a beneficial improvement when on therapy, but clearly demosntrated efficacy on biochemical parameters.

52. Lock, E.A., P. Gaskin, M. Ellis, et al., Tyrosinemia produced by 2-(2-nitro-4trifluoromethylbenzoyl)-cyclohexane-1,3-dione (NTBC) in experimental animals and its relationship to corneal injury. Toxicol Appl Pharmacol, 2006. 215(1): p. 9-16.

53. Holme, E. and S. Lindstedt, Tyrosinaemia type $I$ and NTBC (2-(2-nitro-4trifluoromethylbenzoyl)-1,3-cyclohexanedione). J Inherit Metab Dis, 1998. 21(5): p. 507-17.

54. Masurel-Paulet, A., J. Poggi-Bach, M.O. Rolland, et al., NTBC treatment in tyrosinaemia type I: long-term outcome in French patients. J Inherit Metab Dis, 2008. 31(1): p. 81-7. 
55. Stewart, R.M., M.C. Briggs, J.C. Jarvis, et al., Reversible keratopathy due to hypertyrosinaemia following intermittent low-dose nitisinone in alkaptonuria: a case report. JIMD Rep, 2014. 17: p. 1-6.

56. Suwannarat, P., K. O'Brien, M.B. Perry, et al., Use of nitisinone in patients with alkaptonuria. Metabolism, 2005. 54(6): p. 719-28.

* This paper shows the effects of incremental doses of nitisinone and the interesting outcoem that patients reported reduced pain when on nitisinone.

57. Ranganath, L.R., A.M. Milan, A.T. Hughes, et al., Suitability Of Nitisinone In Alkaptonuria 1 (SONIA 1): an international, multicentre, randomised, open-label, no-treatment controlled, parallel-group, dose-response study to investigate the effect of once daily nitisinone on 24-h urinary homogentisic acid excretion in patients with alkaptonuria after 4 weeks of treatment. Ann Rheum Dis, 2016. 75(2): p. 362-7.

58. Sloboda, N., A. Wiedemann, M. Merten, et al., Efficacy of low dose nitisinone in the management of alkaptonuria. Mol Genet Metab, 2019.

59. Ranganath, L.R., M. Khedr, A.M. Milan, et al., Nitisinone arrests ochronosis and decreases rate of progression of Alkaptonuria: Evaluation of the effect of nitisinone in the United Kingdom National Alkaptonuria Centre. Mol Genet Metab, 2018. 125(1-2): p. 127-134.

60. Taylor, A.M., A.J. Preston, N.K. Paulk, et al., Ochronosis in a murine model of alkaptonuria is synonymous to that in the human condition. Osteoarthritis Cartilage, 2012. 20(8): p. 880-6.

* This manuscript details the simialrities in symptoms of AKU mice and humans, paving the way for follow-up studies of AKU mice and their treatment with nitisinone

61. Keenan, C.M., A.J. Preston, H. Sutherland, et al., Nitisinone Arrests but Does Not Reverse Ochronosis in Alkaptonuric Mice. JIMD Rep, 2015. 24: p. 45-50.

** This paper is the first demonstration of the effect of nitisinone in a model animal system showing how it arrests or inhibits ochronosis in AKU mice.

62. Mistry, J.B., D.J. Jackson, M. Bukhari, and A.M. Taylor, Osteoarticular cells tolerate short-term exposure to nitisinone-implications in alkaptonuria. Clin Rheumatol, 2016. 35(2): p. 513-6.

* This paper is the first to demonstrate that nitisinone has no adverse affect on osteoarticular cells.

63. Mistry, J.B., D.J. Jackson, M. Bukhari, and A.M. Taylor, A role for interleukins in ochronosis in a chondrocyte in vitro model of alkaptonuria. Clin Rheumatol, 2016. 35(7): p. 1849-56.

64. García, M.I., A. de la Parra, C. Arias, et al., Long-term cognitive functioning in individuals with tyrosinemia type 1 treated with nitisinone and protein-restricted diet. Molecular genetics and metabolism reports, 2017. 11: p. 12-16.

65. Honar, N., N. Shakibazad, Z. Serati Shirazi, et al., Neurological Crises after Discontinuation of Nitisinone (NTBC) Treatment in Tyrosinemia. Iranian journal of child neurology, 2017. 11(4): p. 66-70.

66. Hillgartner, M.A., S.B. Coker, A.E. Koenig, et al., Tyrosinemia type I and not treatment with NTBC causes slower learning and altered behavior in mice. J Inherit Metab Dis, 2016. 39(5): p. 673-682.

67. Thimm, E., D. Herebian, B. Assmann, et al., Increase of CSF tyrosine and impaired serotonin turnover in tyrosinemia type I. Mol Genet Metab, 2011. 102(2): p. 122-5.

68. Davison, A.S., N. Strittmatter, H. Sutherland, et al., Assessing the effect of nitisinone induced hypertyrosinaemia on monoamine neurotransmitters in brain tissue from a murine model of alkaptonuria using mass spectrometry imaging. Metabolomics : Official journal of the Metabolomic Society, 2019. 15(5): p. 68-68.

69. White, A. and C.T. M, Nitisinone-Induced Keratopathy in Alkaptonuria: A Challenging Diagnosis Despite Clinical Suspicion. JIMD Rep, 2018. 40: p. 7-9.

70. Khedr, M., S. Judd, M.C. Briggs, et al., Asymptomatic Corneal Keratopathy Secondary to Hypertyrosinaemia Following Low Dose Nitisinone and a Literature Review of Tyrosine Keratopathy in Alkaptonuria. JIMD Rep, 2018. 40: p. 31-37. 
71. Hughes, A.T., A.M. Milan, P. Christensen, et al., Urine homogentisic acid and tyrosine: simultaneous analysis by liquid chromatography tandem mass spectrometry. J Chromatogr B Analyt Technol Biomed Life Sci, 2014. 963: p. 106-12.

* This paper demonstrates the validity and accuracy of reverse phase LC-MS/MS for quantifying urinary HGA and Tyrosine in AKU, enbaling clincial monitoring of pre-and post nitinsone patient profiles.

72. DevelopAKUre. SONIA 2 and DevelopAKUre - Results. http://www.developakure.eu/Results 2019 29th August 2019].

73. Taylor, A.M., D.D. Jenks, V.D. Kammath, et al., Raman Spectroscopy identifies differences in ochronotic and non-ochronotic cartilage; a potential novel technique for monitoring ochronosis. Osteoarthritis Cartilage, 2019.

** This paper is the first to demonstrate the potential application of a non-invasive technique which could potentially monitor ochronosis in vivo. It shows clear spectral differenes between ochronotic and non-ochronotic cartilages enabling deicsions about timed administration of any therapy to be patient specific, in the future

74. Buckley, K.K., J; Gikas, PD; et al, Measurement of abnormal bone composition in vivo using noninvasive Raman spectroscopy. IBMS BoneKEy, 2014. 11: p. 1-3.

75. Buckley, K., J.G. Kerns, J. Vinton, et al., Towards the in vivo prediction of fragility fractures with Raman spectroscopy. J Raman Spectrosc, 2015. 46(7): p. 610-618.

76. Ranganath, L.R. and T.F. Cox, Natural history of alkaptonuria revisited: analyses based on scoring systems. J Inherit Metab Dis, 2011. 34(6): p. 1141-51.

77. Langford, B., M. Besford, A. Hall, et al., Alkaptonuria Severity Score Index Revisited: Analysing the AKUSSI and Its Subcomponent Features. JIMD Rep, 2018. 41: p. 53-62.

78. Griffin, R., E.E. Psarelli, T.F. Cox, et al., Data on items of AKUSSI in Alkaptonuria collected over three years from the United Kingdom National Alkaptonuria Centre and the impact of nitisinone. Data Brief, 2018. 20: p. 1620-1628. 